\title{
SIMULATING EPITAXIAL TEXTURES
}

\author{
FRANCIS WAGNER*, LAURENCE LAMM* and DIDIER DEPARIS** \\ *LM2P/ISGMP, Université de Metz, Ile du Saulcy, 57045 Metz-Cedex, France, \\ **SOLLAC/LEDEPP, Allée des Tilleuls, 57 Florange, France
}

(Received 17 March 1995)

\begin{abstract}
The way of calculating the texture of a polycrystalline deposit over a polycrystalline substrate is considered in this paper. The proposed procedure consists in taking into account the epitaxial relationships and in defining some kind of selection rules according to the assumed crystallographic surface state of the substrate. To illustrate the interest of such a procedure, textures are calculated under various conditions and compared with 'experimental' textures for electrodeposited zinc deposits over steel sheets.
\end{abstract}

KEY WORDS: Epitaxy, deposit, texture, zinc electrodeposition.

\section{INTRODUCTION}

The use of multi-materials in order to optimize physical or mechanical properties is a trend in today's technology. There are many processes which allow to deposit more or less thin films on a given substrate (CVD, PVD, galvanization processes for example). In the case of polycrystalline materials the distribution of orientations and misorientations is known to influence various properties of the materials. According to the conditions of the deposition process, epitaxial relationships can occur between the substrate and the deposit and therefore determine partially or fully the crystallographic texture of the deposit. Under the name epitaxial texture we understand the following situation: at the surface of a grain of the substrate, considered as a small monocrystal, 3D nuclei could appear whose orientations are correlated with the orientation of the substrate grain and will be maintained during growth (Volmer-Weber mode of growth) (Pashley, 1991); considering a statistically relevant population of substrate grains, this will give rise to a crystallographic texture in the deposit correlated with the substrate texture.

Epitaxial relationships are usually described by indicating planes and directions which are parallel in the substrate and the deposit i.e. (hkl) $)_{\text {sub }} / /\left(h^{\prime} \mathbf{k}^{\prime} \mathrm{l}^{\prime}\right)_{\mathrm{dep}}$ and $[\mathrm{uvw}]_{\text {sub }} / /\left[\mathrm{u}^{\prime} \mathbf{v}^{\prime} \mathrm{w}^{\prime}\right]_{\text {dep }}$. In a quite equivalent way one can specify the rotation $\Delta \mathrm{g}$ between an orientation in the substrate and an orientation in the deposit related to the first one throug epitaxy.

Because of the crystal symmetry of the substrate and according to the particular crystallographic relationships, $\mathrm{N}$ different orientations are possible for the deposit growing on a given grain of the substrate. This number $\mathbf{N}$ of orientations can be calculated in a quite similar way as in the case of phase transformation (Humbert et al., 1992). There is then a set of rotations $\{\Delta$ gi; $1 \leq \mathrm{i} \leq \mathrm{N}\}$ which allow to calculate the possible orientations $\mathbf{g}_{\mathrm{i}}$ of a new grain: 


$$
\mathrm{g}_{\mathrm{i}}^{\prime}=\Delta \mathrm{gi} \cdot \mathrm{g}
$$

For example for a cubic substrate and an hexagonal deposit and for the epitaxy relationship $(110)_{\text {sub }} / /(00.1)_{\text {dep }}$ and $[111]_{\text {sub }} / /[11.0]_{\text {dep }}$, which will be considered later on, $\mathrm{N}$ is equal to 12 . In fact only a part of these possible orientations usually appear. When both the substrate and the deposit are polycrystalline it is not an easy task to recognize if the crystallographic texture of the deposit is correlated through epitaxial relationships with the texture of the substrate.

A program allowing to calculate the deposit texture from the substrate texture for a given epitaxy relationship could be very useful to answer this question. The comparison of simulated and real deposit textures would indeed be a way to handle the problem. Moreover such a comparison could help to find more precisely the rules governing the development of orientations in the deposit. It is the purpose of the present paper to define a way of simulating epitaxial textures.

\section{DISCRETIZATION OF THE TEXTURE FUNCTIONS}

The surface texture of the substrate is usually described by a continuous function, the O.D.F. $f_{s}(g)$, which has the crystal symmetry of the considered substrate material. The function $f_{s}(\Delta g . g)$ would then correspond to the O.D.F., $f_{d}(g)$, of a deposit texture correlated with the substrate texture through an epitaxial relationship characterized by $\Delta g$. However with such a simple calculation of $f_{d}(g)$, all the possible new orientations appear with an equal probability in the deposit texture. If one wants to restrict or select the number of new orientations it is necessary, either to introduce in the calculation a selection function in a quite similar way as it is done in phase transformation problems (Bunge et al., 1983; Humbert, 1987), or to discretize the starting texture function, $f_{s}(g)$, in order to have the possibility to control the number of the considered new orientations for each initial substrate orientation.

The discretization of an O.D.F. $\mathrm{f}(\mathrm{g})$ is performed by considering that the orientation space (the so-called Euler space when $g$ is described with the three Euler angles) is divided into $K$ small boxes centered on rotations $g_{k}$; the texture consists then in a set of $K$ orientations or 'grains' $\left\{g_{k}, w_{k} ; 1 \leq k \leq K\right\}$ where $w_{k}$ is the weight attributed to the grain number $k$. $w_{k}$ is calculated by integrating $f(g)$ over the volume $b_{k}$ of the box number $k$ :

$$
w_{k}=\int_{b_{k}} f(g) d g
$$

If a texture is described with such a dicrete set of 'grains' $\left\{g_{k}, w_{k} ; 1 \leq k \leq K\right\}$ it is possible to come back to a continuous function $\mathrm{f}^{\mathrm{r}}(\mathrm{g})$ by considering that each orientation consists in a gaussian peak and by adding all these elementar functions:

$$
\mathrm{f}^{\mathrm{r}}(\mathrm{g})=\sum_{\mathbf{k}} \mathrm{w}_{\mathbf{k}} \mathrm{f}^{\mathrm{G}}\left(\mathrm{g}_{\mathrm{k}}, \mathrm{h}_{\mathrm{k}}, \mathrm{g}\right)
$$

where $f^{G}\left(g_{k}, h_{k}, g\right)$ is a gaussian peak centered at $g_{k}$ with the full half width $h_{k}$. Such a way to handle individual orientations is very often used when calculating O.D.F.'s from individual orientation measurements or from orientation calculations by using models of polycrystalline plasticity. A difficult point is to define the half width $h_{k}$ of the gaussian peaks (Wagner et al., 1981; Engler et al., 1994).

Figure 1 shows some sections $\left(\varphi_{1}=0^{\circ}\right.$ and $\left.\varphi_{2}=45^{\circ}\right)$ of an 'experimental' O.D.F., 

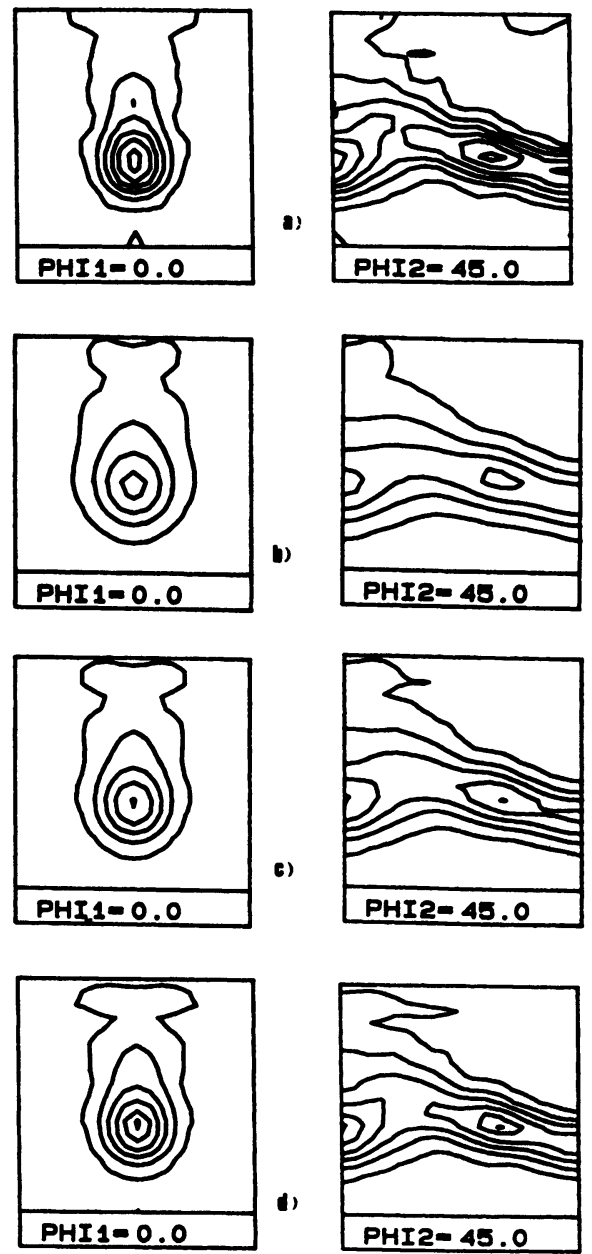

Figure 1 Two O.D.F. sections for a) a low carbon steel and for recalculated functions after dicretization and superimposition of gaussian peaks with $b$ ) $\left.b=11.7^{\circ}, c\right) b=6.3^{\circ}$ and $d$ ) $b=5.0^{\circ}$; levels: $1.0,2.5,4.0,5.5,7.0,8.0$.

$\mathrm{f}(\mathrm{g})$, of a low carbon steel calculated from pole figure measurements by using the harmonic method including the positivity technique (Dahms and Bunge, 1988; Wagner et al., 1990). Three 'recalculated' O.D.F.'s $\mathrm{f}^{\mathrm{r}}(\mathrm{g})$, obtained after discretization of $\mathrm{f}(\mathrm{g})$ and recalculation of a continuous function under different conditions are also represented; the number $\mathrm{K}$ of 'grains', in this example, is 5832 (boxes of $5^{\circ}$ in the Euler space) and the full half width $h_{k}$ for gaussian peaks is set respectively to $11.7^{\circ}$, $8.3^{\circ}$ and $5.0^{\circ}$. By comparing 'experimental' and 'recalculated' functions the trend clearly appears: the main features are present in the 'recalculated' O.D.F.'s but the texture is weaker especially with the largest full half widths for the gaussian peaks. Table I below contains the maximal and minimal values of these O.D.F.'s. 
Table I maximal and minimal values in an 'experimental' O.D.F. and in 'recalculated' O.D.F.'s obtained after discretization and superimposition of gaussian peaks.

\begin{tabular}{lcccc}
\hline & 'exp.' $f(g)$ & $f^{\prime}(g)$ with $h_{k}=11.7^{\circ}$ & $f^{\prime}(g)$ with $h_{k}=8.3^{\circ}$ & $f^{\prime}(g)$ with $h_{k}=5.0^{\circ}$ \\
\hline maximum & 9.15 & 6.03 & 7.11 & 8.15 \\
minimum & -0.28 & 0.05 & 0.01 & -0.19 \\
\hline
\end{tabular}

Even with the positivity technique, small negative values can appear in the 'experimental' O.D.F., mainly because of the errors and incompatibilities in the pole figure data. For the recalculated O.D.F.'s, small negative values are linked with the truncation error (the maximal rank of the series expansions, Imax, has been set to 34 in all the cases).

From Figure 1 and Table $I$ it appears nevertheless that the texture is not too much altered by using this technique of discretization (and recalculation of a continuous function with an adequate $h_{k}$ parameter)

If such a discretization is applied to a substrate texture, the calculation of the deposit texture correlated through an epitaxial relationship is then reduced to the question of defining selection rules for the generation of deposit orientations on a given substrate grain.

\section{SELECTION RULES}

For a given epitaxial relationship (hkl) $)_{\text {sub }} / /\left(h^{\prime} \mathbf{k}^{\prime} \mathrm{l}^{\prime}\right)_{\text {dep }}$ and $[\mathrm{uvw}]_{\text {sub }} / /\left[\mathrm{u}^{\prime} \mathrm{v}^{\prime} \mathrm{w}^{\prime}\right]_{\text {dep }}$ and for a given grain $\left[g_{k}, w_{k}\right]$ of the substrate, it is possible to calculate the orientation of all the symmetrycally equivalent planes $(\mathrm{hkl})_{\text {sub }}$ and all the symmetrically equivalent $[\mathrm{uvw}]_{\text {sub }}$ directions in the specimen coordinate system. A selection rule consists then in assessing which (hkl) sub among all the symmetrically equivalent ones and which $[\mathrm{uvw}]_{\text {sub }}$ determine the orientations of deposit grains. This requires to know the crystallographic nature of the surface of each grain, which is of course very seldomly the case. Reasonable assumptions have thus to be made about this geometry of the surface.

Let us consider, for sake of simplicity, that the $\{h k l\}_{\text {sub }}$ planes involved in the epitaxy relationship are the most close packed planes and that they limit the surfaces of the grains. For 'flat surfaces' those $\{h k l\}_{\text {sub }}$ planes which make the smallest angle with the 'mean surface' will be present. On the contrary if facets exist at the surface, $\{h k l\}_{\text {sub }}$ planes with large angle versus the 'mean surface' will be present.

A selection rule can therefore be based on the angle $\theta_{\mathrm{j}}$ of the $\{\mathrm{hkl}\}_{\text {sub }}$ planes versus the mean surface in a given grain whose orientation $g_{k}$ is known; $j$ runs between 1 and $J$ the number of symetrically equivalent $\{h k l\}_{\text {sub }}$ planes. $\theta_{j}$ is obtained by calculating:

$$
\cos \left(\theta_{j}\right)=\left(g_{k} h_{j}\right) \cdot C
$$

where $h_{j}$ is a unit vector normal to the $(\mathbf{h k l})_{\text {sub }}$ plane number $\mathrm{j}$ in the crystal coordinate system and $\mathbf{C}$ is the unit vector along the normal direction in the specimen coordinate system. For a given criterion, one will then select $\mathrm{P}(\mathrm{hkl})_{\text {sub }}$ planes. Remark that $\mathrm{P}$ can be different from one substrate grain to another one because, according to the 
orientation $\mathrm{g}_{\mathrm{k}}$, more or less (hkl) $)_{\text {sub }}$ planes can satisfy a criterion such a minimal angle versus the 'mean surface' for example.

Assume that there are several symmetrically equivalent [uvw $]_{\text {sub }}$ directions in one (hkl) sub plane. Once a (hkl) sub plane has been selected, one has to choose one or several [hkl] $]_{\text {ub }}$ directions in this plane so that the orientations of the deposit grains can be defined. No evident criterion appears for selecting [uvw] $]_{\text {sub }}$ directions: the most reasonable choices seem to be either one arbitrary [uvw] sub direction or all the $[\mathrm{uvw}]_{\text {sub }}$ directions in the plane. If one calls $\mathrm{D}$ this number of directions, each grain of the substrate will give rise to PxD orientations of the deposit; these orientations are assumed to have the same volume $w_{k} /(\operatorname{PxD})$ where $w_{k}$ is the volume of the substrate starting grain.

By considering all the grains of the substrate, such a procedure results lastly in a set $\left\{g_{k l}^{\prime}, w_{k l}^{\prime}\right\}$ of orientations; 1 numbers the deposit orientations deduced from the substrate grain number $k$ and runs from 1 up to a value $L(k)$ which depends on $k$. The number $\mathbf{N}$ ' of deposit 'grains' or orientations is therefore very large:

$$
\mathbf{N}^{\prime}=\sum_{\mathbf{k}} \mathbf{L}(\mathbf{k})
$$

Practically, in order to save computer time, the end orientation space is divided into $M$ small boxes and the weight allocated to each box is calculated by adding the weights $w_{k 1}^{\prime}$ of the orientations $g_{k 1}^{\prime}$ falling in this box. The deposit texture consists then in a set $\left\{g_{m}^{\prime}, w_{m}^{\prime} ; 1 \leq m \leq M\right\}$ of individual orientations from which $a$ continuous function can be calculated.

\section{EXAMPLE}

To illustrate the proposed way of simulating epitaxy textures the previously described procedure has been applied to calculate zinc deposit textures on a low carbon steel sheet. Real zinc deposit textures are approximatively fibre textures. According to the parameters of the electrodeposition process various $\mathrm{Zn}$ textures can occur (Caillet, 1992; De Boeck et al., 1993). Roughly one can consider four texture types:

- type A consists in a fiber <11.0>//ND (ND is the specimen normal); this means maxima in the Euler space at $\Phi=90^{\circ}, \varphi_{2}=0^{\circ}\left(\equiv 60^{\circ}\right) \forall \varphi_{1}$

- type $B$ consists in a fiber $<10.3>/ / N D$; this means maxima in the Euler space at $\Phi=35^{\circ}, \varphi_{2}=0^{\circ}\left(\equiv 60^{\circ}\right) \forall \varphi_{1}$

- type $C$ consists in a fiber with $(00.1) / / y=\left\{\alpha=30^{\circ}, \beta=0^{\circ}\right)$ where $\alpha$ and $\beta$ are the spherical angular coordinates of the specimen direction $y$. This means maxima in the Euler space at $\varphi_{1}=90^{\circ}, \Phi=30^{\circ}, \forall \varphi_{2}$

- type $\mathrm{D}$ consists in a fiber with $<00.1>/ / \mathrm{ND}$; this means maxima in the Euler space at $\Phi=0^{\circ}, \forall \varphi_{1}$ and $\forall \varphi_{2}$

This is illustrated at Figure 2 in particular O.D.F. sections for $10 \mu \mathrm{m}$ thick zinc deposits on steel sheets produced under various conditions either on a laboratory electrodeposition line or on industrial lines. $\mathrm{Zn}$ textures can also be a mixture of these texture types, mainly in the case of type B and C textures (see Figure $2 \mathrm{~b}$ )).

As already mentioned, an epitaxy relationship exists between $\mathrm{Fe}_{\alpha}$ and $\mathrm{Zn}$ which reads $(110)_{\text {sub }} / /(00.1)_{\text {dep }}$ and $[111]_{\text {sub }} / /[11.0]_{\text {dep }}$. When considering all the $\{110\}$ and 
a)

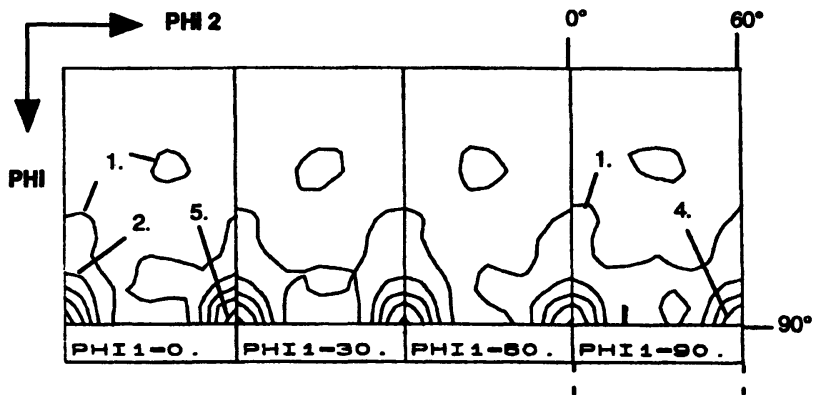

b)

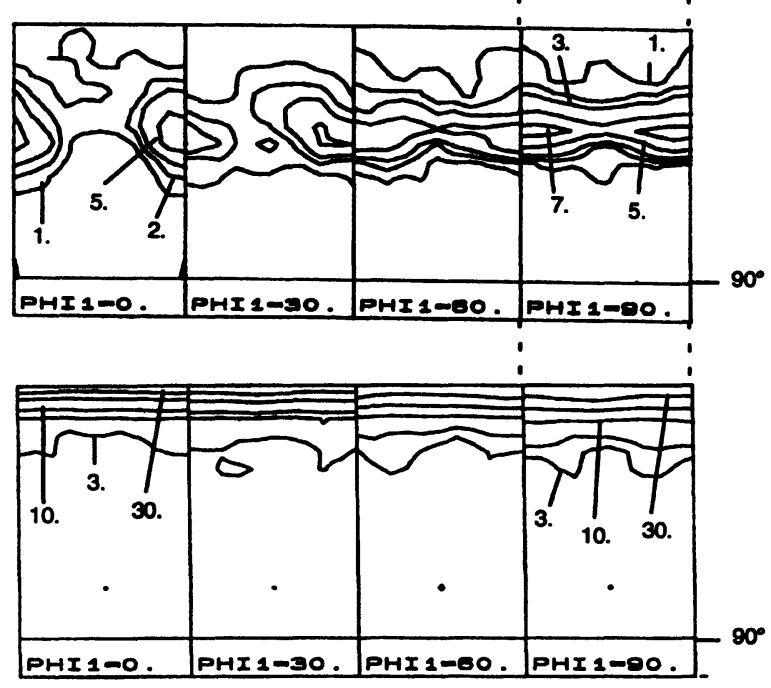

Figure 2 O.D.F. sections of electrodeposited zinc corresponding to a) type A texture; b) type B and $\mathrm{C}$ textures; c) type $\mathrm{D}$ texture.

$<111>$ symetrically equivalent planes and directions, 12 distinct $\mathrm{Zn}$ orientations are obtained. In a given (110) plane, there are two different $\langle 111\rangle$ directions so that, when $(00.1)$ is parallel to $(110)$, two $\mathrm{Zn}$ orientations can appear $(\mathrm{D}=2)$ according to the direction $<111>$ to which [11.0] is parallel.

Epitaxy textures have been simulated by using this epitaxy relationship and by using the steel texture presented at Figure 1. Several conditions of simulation have been used, namely

- S1: all the 12 possible orientations are generated for each steel 'grain'

- S21: only the (110) planes with the minimal angle $\theta$ with the surface and 1 direction [111] in this plane are considered for epitaxy in each steel 'grain'

- S22: only the (110) planes with the minimal angle $\theta$ with the surface and both directions [111] in this plane are considered for epitaxy in each steel 'grain'

- S31: only the (110) planes with the maximal angle $\theta$ with the surface and 1 direction [111] in this plane are considered for epitaxy in each steel 'grain'

- S32: only the (110) planes with the maximal angle $\theta$ with the surface and both directions [111] in this plane are considered for epitaxy in each steel 'grain' 
Some O.D.F. sections of these calculated textures are shown at Figure 3.

By comparing these calculated textures with the real ones, some conclusions can be drawn. First it is quite clear that no calculated texture is similar with the 'experimental' type D texture (Figure 2c) so that the previously suggested mechanisms

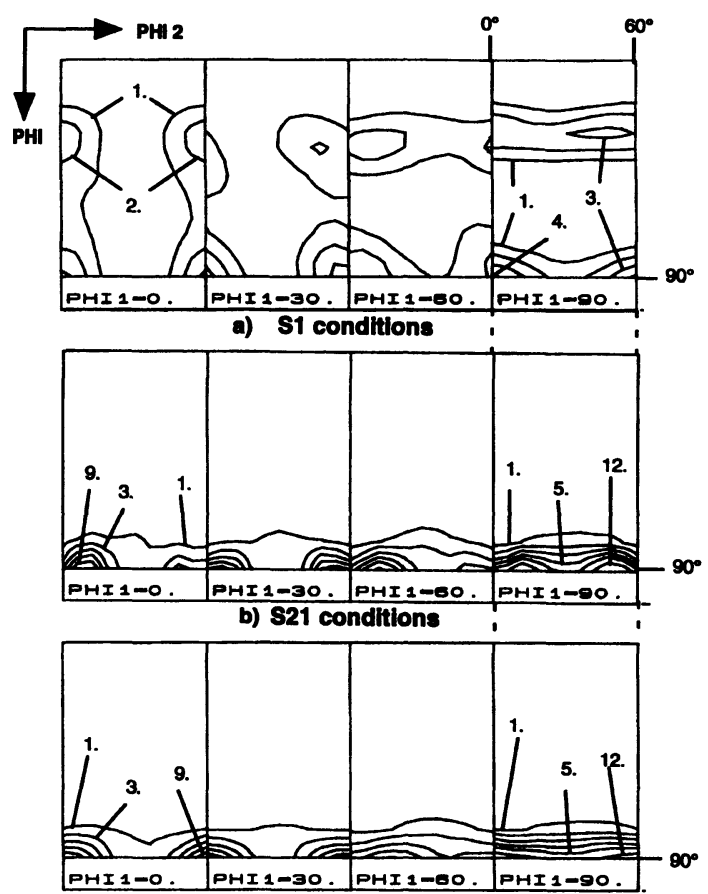

c) $\mathbf{S 2 2}$ conditions

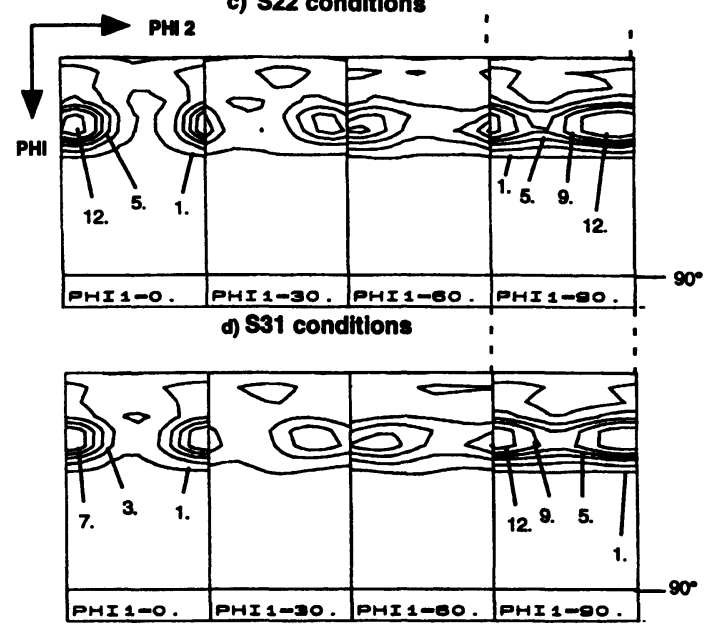

•) $\mathbf{S 3 2}$ conditions

Figure 3 O.D.F. sections of calculated textures under various assumptions for zinc deposits a) S1 conditions; b) S21 conditions; c) S22 conditions d) S31 conditions; e) S32 conditions. 
for the formation of deposit texture are not valid in such a case. The factors which prevent the formation of an epitaxial texture are only partially understood (Caillet, 1992; De Boeck et al., 1993). The calculated textures show more or less similarities with the experimental textures of type A, B and C. Nevertheless the calculated texture with $\mathrm{S} 1$ conditions (each steel orientation generates 12 zinc orientations) is too weak when compared with the 'experimental' textures. Both calculated textures with conditions S22 and S32 show preferred orientations corresponding to those of the 'experimental' textures except that the dispersion is higher for these last ones. Calculated textures with S21 and S31 conditions are slightly less realistic with sometimes small shifts of the peaks always in comparison to the 'experimental' texture.

This agreement between calculated textures (mainly under conditions S22 and S32) and experimental ones speaks for the fact that in many cases the deposit texture is determined by the existing texture of the substrate together with a selection depending on the surface state of the substrate grains. A complete and indisputable demonstration would require individual orientation measurements of both substrate and deposit grains together with the caraterization of the crystallographic plane at the interface, which could be carried out by using EBSD.

\section{CONCLUSION}

The formation of the texture of a polycrystalline deposit can be strongly influenced by the texture of the polycrystalline substrate according to the parameters of the used deposition process. Epitaxial relationships between deposit and substrate grains is a possible explanation when such a texture correlation occurs. It is therefore important to recognize if the deposit texture can be deduced from the substrate one. This requires not only to know the epitaxial crystallographic relationship but also to define selection rules describing those deposit orientations, among the possible ones, which effectively develop.

The proposed procedure to calculate epitaxial textures is then an interesting tool to improve the understanding of texture formation in deposits.

\section{References}

Bunge, H. J., Humbert, M. and Welch, P. I. (1983) Scripta Met., 17, p. 1403-1405.

Caillet D. (1992) Ph. D., University of Metz (France).

Dahms, M. and Bunge, H. J. (1988) Textures and Microstructures, 10, p. 21-35.

De Boeck, A., Vanthournout, M. and Van der Hoeven (1994) proceedings of the IDDRG 18th congress, Lisbon, Portugal, ed. INETI, p. 55-65.

Engler, O., Gottstein G., Pospiech, J. and Jura, J. (1994) proceedings of the 10th ICOTOM, Trans Tech Publications, p. 259-273.

Humbert, M. (1987) These de doctorat d'Etat, University of Metz (France).

Humbert, M., Wagner F. and Esling C. (1992) J. Appl. Cryst., 25, p. 724-730.

Pashley, D. W. (1991) Processing of metals and alloys, 15, p. 289-320.

Wagner, F., Wenk, H. R., Esling, C. and Bunge, H. J. (1981) Phys. Stat. Sol. (a), 76, p. 269-285.

Wagner, F., Humbert, M., Muller, J. and Esling C. (1990) Europhys. Lett., 11(5), p. 479-483. 